PDES, SUBMANIFOLDS AND

AFFINE DIFFERENTIAL GEOMETRY

BANACH CENTER PUBLICATIONS, VOLUME 69

INSTITUTE OF MATHEMATICS

POLISH ACADEMY OF SCIENCES

WARSZAWA 2005

\title{
THE GAPS IN THE SPECTRUM OF THE SCHRÖDINGER OPERATOR
}

\author{
HAIZHONG LI and LINLIN SU \\ Department of Mathematical Sciences, Tsinghua University \\ Beijing, 100084, People's Republic of China \\ E-mail: hli@math.tsinghua.edu.cn
}

\begin{abstract}
We obtain inequalities between the eigenvalues of the Schrödinger operator on a compact domain $\Omega$ of a submanifold $M$ in $R^{N}$ with boundary $\partial \Omega$, which generalize many existing inequalities for the Laplacian on a bounded domain of a Euclidean space. We also establish similar inequalities for a closed minimal submanifold in the unit sphere, which generalize and improve Yang-Yau's result.
\end{abstract}

1. Introduction. Let $x: M \rightarrow R^{N}$ be an $m$-dimensional submanifold in $N$-dimensional Euclidean space with the mean curvature vector $\mathbf{H}$. Let $\Omega \subset M$ be a compact domain on $M$ with boundary $\partial \Omega$. We consider the Schrödinger operator

$$
L=\Delta-V
$$

where $V$ is a nonnegative smooth function on $\bar{\Omega}$. We shall consider the following Dirichlet eigenvalue problem of the Schrödinger operator $L$ on $\Omega$ :

$$
\left\{\begin{array}{l}
L u=-\lambda u \quad \text { on } \Omega \\
\left.u\right|_{\partial \Omega}=0
\end{array}\right.
$$

As is well-known, the eigenvalues $\left\{\lambda_{k}\right\}$ of (1.2) are nonnegative, and can be arranged in nondecreasing order as follows:

$$
0<\lambda_{1} \leq \lambda_{2} \leq \cdots \leq \lambda_{n} \leq \cdots
$$

Assume we know the first $n$ eigenfunctions of $L$

$$
u_{1}, u_{2}, \ldots, u_{n}
$$

2000 Mathematics Subject Classification: Primary 35P15; Secondary 58G25.

Key words and phrases: eigenvalues of the Schroedinger operator, inequality, bounded domain of submanifold, mean curvature.

This work is partially supported by the grant No. 10131020 of NSFC.

The paper is in final form and no version of it will be published elsewhere. 
i.e., $L u_{i}=-\lambda_{i} u_{i}$, having the properties

$$
\begin{gathered}
u_{i}=0 \quad \text { on } \partial \Omega, \\
\int_{\Omega} u_{i} u_{j}=\left\{\begin{array}{lll}
1, & \text { when } & i=j, \\
0, & \text { when } & i \neq j .
\end{array}\right.
\end{gathered}
$$

To obtain information about the next eigenvalue, we shall need some appropriate "trial functions". In fact, following [5], we can use the following trial functions

$$
\phi_{1}^{\alpha}, \phi_{2}^{\alpha}, \ldots, \phi_{n}^{\alpha} .
$$

Any one of these trial functions, say $\phi_{i}^{\alpha}$, must satisfy two conditions:

$$
\phi_{i}^{\alpha}=0 \quad \text { on } \quad \partial \Omega
$$

$$
\int_{\Omega} \phi_{i}^{\alpha} u_{j}=0
$$

where $i, j=1,2, \ldots, n$. We choose (following [5])

$$
\phi_{i}^{\alpha}=x_{\alpha} u_{i}-\sum_{j=1}^{n} a_{i j}^{\alpha} u_{j}, \quad \alpha=1, \ldots, N,
$$

where

$$
a_{i j}^{\alpha}=\int_{\Omega} x_{\alpha} u_{i} u_{j}=a_{j i}^{\alpha}, \quad x(p)=\left(x_{1}(p), \ldots, x_{N}(p)\right) \in R^{N}, \quad \forall p \in \Omega .
$$

It is easy to see that the functions $\phi_{i}^{\alpha}$ satisfy (1.5) and (1.6), i.e., $\phi_{i}^{\alpha}$ are all orthogonal to $u_{1}, \ldots, u_{n}$ and vanish at the boundary. We also have, by use of (1.7) and (1.6),

$$
\begin{gathered}
\int_{\Omega}\left(\phi_{i}^{\alpha}\right)^{2}=\int_{\Omega} \phi_{i}^{\alpha} x_{\alpha} u_{i}=\int_{\Omega} x_{\alpha}^{2} u_{i}^{2}-\sum_{j=1}^{n}\left(a_{i j}^{\alpha}\right)^{2} \\
-\Delta \phi_{i}^{\alpha}=-u_{i} \Delta x_{\alpha}-2 \nabla u_{i} \cdot \nabla x_{\alpha}+\lambda_{i} x_{\alpha} u_{i}-\sum_{j=1}^{n} a_{i j}^{\alpha} \lambda_{j} u_{j}-V \phi_{i}^{\alpha} \\
-\int_{\Omega} \phi_{i}^{\alpha} L\left(\phi_{i}^{\alpha}\right)=\int_{\Omega} \phi_{i}^{\alpha}\left(-\Delta \phi_{i}^{\alpha}\right)+\int_{\Omega} V\left(\phi_{i}^{\alpha}\right)^{2} \\
=-\int_{\Omega} \phi_{i}^{\alpha} \Delta x_{\alpha} \cdot u_{i}-2 \int_{\Omega} \phi_{i}^{\alpha} \nabla u_{i} \cdot \nabla x_{\alpha}+\lambda_{i} \int_{\Omega}\left(\phi_{i}^{\alpha}\right)^{2} .
\end{gathered}
$$

Hence by the variational principle for $\lambda_{n+1}$, we have

$$
\lambda_{n+1} \int_{\Omega}\left(\phi_{i}^{\alpha}\right)^{2} \leq-\int_{\Omega} \phi_{i}^{\alpha} L\left(\phi_{i}^{\alpha}\right)=-\int_{\Omega} \phi_{i}^{\alpha} \Delta x_{\alpha} \cdot u_{i}-2 \int_{\Omega} \phi_{i}^{\alpha} \nabla u_{i} \cdot \nabla x_{\alpha}+\lambda_{i} \int_{\Omega}\left(\phi_{i}^{\alpha}\right)^{2},
$$

that is,

$$
0 \leq\left(\lambda_{n+1}-\lambda_{i}\right) \int_{\Omega}\left(\phi_{i}^{\alpha}\right)^{2} \leq-\int_{\Omega} \phi_{i}^{\alpha} \Delta x_{\alpha} \cdot u_{i}-2 \int_{\Omega} \phi_{i}^{\alpha} \nabla u_{i} \cdot \nabla x_{\alpha} .
$$

From (1.12), by use of (1.6) we get

$$
\left(\lambda_{n+1}-\lambda_{i}\right) \int_{\Omega}\left(\phi_{i}^{\alpha}\right)^{2} \cdot\left[-\int_{\Omega} \phi_{i}^{\alpha} \Delta x_{\alpha} \cdot u_{i}-2 \int_{\Omega} \phi_{i}^{\alpha} \nabla u_{i} \cdot \nabla x_{\alpha}\right]
$$




$$
\begin{aligned}
& \leq\left[-\int_{\Omega} \phi_{i}^{\alpha} \Delta x_{\alpha} \cdot u_{i}-2 \int_{\Omega} \phi_{i}^{\alpha} \nabla u_{i} \cdot \nabla x_{\alpha}\right]^{2}=\left[\int_{\Omega} \phi_{i}^{\alpha}\left(\Delta x_{\alpha} \cdot u_{i}+2 \nabla u_{i} \cdot \nabla x_{\alpha}\right)\right]^{2} \\
& =\left[\int_{\Omega} \phi_{i}^{\alpha}\left(\Delta x_{\alpha} \cdot u_{i}+2 \nabla u_{i} \cdot \nabla x_{\alpha}-2 \sum_{j=1}^{n} b_{i j}^{\alpha} u_{j}\right)\right]^{2} .
\end{aligned}
$$

Define

$$
\begin{gathered}
I_{\alpha i}=\int_{\Omega}\left|\nabla x_{\alpha}\right|^{2} u_{i}^{2}, \\
b_{i j}^{\alpha}=\int_{\Omega} u_{j} \nabla x_{\alpha} \cdot \nabla u_{i}+\frac{1}{2} \int_{\Omega} \Delta x_{\alpha} \cdot u_{i} u_{j},
\end{gathered}
$$

By use of the Stokes formula and (1.3), it is easy to check

$$
b_{i j}^{\alpha}=-b_{j i}^{\alpha}, \quad \alpha=1,2, \ldots, N ; \quad i, j=1,2, \cdots, n .
$$

We have

$$
\begin{gathered}
-2 \int_{\Omega} \phi_{i}^{\alpha} \nabla x_{\alpha} \cdot \nabla u_{i}=-\int_{\Omega} x_{\alpha} \nabla x_{\alpha} \cdot \nabla\left(u_{i}^{2}\right)+2 \sum_{j=1}^{n} a_{i j}^{\alpha} \int_{\Omega} u_{j} \nabla x_{\alpha} \cdot \nabla u_{i} \\
=I_{\alpha i}+\int_{\Omega} u_{i}^{2} x_{\alpha} \Delta x_{\alpha}+2 \sum_{j=1}^{n} a_{i j}^{\alpha} \int_{\Omega} u_{j} \nabla x_{\alpha} \cdot \nabla u_{i} \\
-\int_{\Omega} u_{i} \phi_{i}^{\alpha} \Delta x_{\alpha}-2 \int_{\Omega} \phi_{i}^{\alpha} \nabla u_{i} \cdot \nabla x_{\alpha}=I_{\alpha i}+2 \sum_{j=1}^{n} a_{i j}^{\alpha} b_{i j}^{\alpha} .
\end{gathered}
$$

From the definition of $a_{i j}^{\alpha}$,

$$
\begin{aligned}
\lambda_{i} a_{i j}^{\alpha} & =\int_{\Omega}\left(-L u_{i}\right) x_{\alpha} u_{j}=\int_{\Omega}\left(-\Delta u_{i}\right) x_{\alpha} u_{j}+\int_{\Omega} V u_{i} u_{j} x_{\alpha} \\
& =\int_{\Omega} u_{i} \Delta\left(-x_{\alpha} u_{j}\right)+\int_{\Omega} V u_{i} u_{j} x_{\alpha} \\
& =-\int_{\Omega} u_{i} u_{j} \Delta x_{\alpha}-2 \int_{\Omega} u_{i} \nabla x_{\alpha} \cdot \nabla u_{j}+\lambda_{j} \int_{\Omega} u_{i} u_{j} x_{\alpha} \\
& =-2 b_{j i}^{\alpha}+\lambda_{j} a_{i j}^{\alpha}=2 b_{i j}^{\alpha}+\lambda_{j} a_{i j}^{\alpha},
\end{aligned}
$$

that is,

$$
2 b_{i j}^{\alpha}=\left(\lambda_{i}-\lambda_{j}\right) a_{i j}^{\alpha} .
$$

Putting (1.17) and (1.18) into (1.13), we obtain for any real number $\epsilon>0$

$$
\begin{aligned}
\left(\lambda_{n+1}-\lambda_{i}\right) \int_{\Omega}\left(\phi_{i}^{\alpha}\right)^{2} \cdot\left[I_{\alpha i}\right. & \left.+\sum_{j=1}^{n}\left(\lambda_{i}-\lambda_{j}\right)\left(a_{i j}^{\alpha}\right)^{2}\right] \\
\leq & {\left[\int_{\Omega} \phi_{i}^{\alpha}\left(\Delta x_{\alpha} \cdot u_{i}+2 \nabla u_{i} \cdot \nabla x_{\alpha}-2 \sum_{j=1}^{n} b_{i j}^{\alpha} u_{j}\right)\right]^{2} } \\
\leq & \int_{\Omega}\left(\phi_{i}^{\alpha}\right)^{2} \cdot \int_{\Omega}\left[\Delta x_{\alpha} \cdot u_{i}+2 \nabla u_{i} \cdot \nabla x_{\alpha}-2 \sum_{j=1}^{n} b_{i j}^{\alpha} u_{j}\right]^{2}
\end{aligned}
$$




$$
\begin{aligned}
& =\int_{\Omega}\left(\phi_{i}^{\alpha}\right)^{2} \cdot\left[\int_{\Omega}\left(\Delta x_{\alpha} u_{i}+2 \nabla u_{i} \cdot \nabla x_{\alpha}\right)^{2}-4 \sum_{j=1}^{n}\left(b_{i j}^{\alpha}\right)^{2}\right] \\
& \leq \int_{\Omega}\left(\phi_{i}^{\alpha}\right)^{2} \cdot\left[(1+1 / \epsilon) \int_{\Omega}\left(\Delta x_{\alpha}\right)^{2} u_{i}^{2}+4(1+\epsilon) \int_{\Omega}\left|\nabla u_{i} \cdot \nabla x_{\alpha}\right|^{2}-4 \sum_{j=1}^{n}\left(b_{i j}^{\alpha}\right)^{2}\right],
\end{aligned}
$$

where we used the Schwarz inequality in the last step.

Dividing by $\int_{\Omega}\left(\phi_{i}^{\alpha}\right)^{2}$, we have for any real number $\epsilon>0$

$$
\begin{aligned}
& \left(\lambda_{n+1}-\lambda_{i}\right)\left[I_{\alpha i}+\sum_{j=1}^{n}\left(\lambda_{i}-\lambda_{j}\right)\left(a_{i j}^{\alpha}\right)^{2}\right] \\
& \leq(1+1 / \epsilon) \int_{\Omega}\left(\Delta x_{\alpha}\right)^{2} u_{i}^{2}+4(1+\epsilon) \int_{\Omega}\left|\nabla u_{i} \cdot \nabla x_{\alpha}\right|^{2}-4 \sum_{j=1}^{n}\left(b_{i j}^{\alpha}\right)^{2} .
\end{aligned}
$$

Inequality (1.20) holds even in the case that $\phi_{i}^{\alpha} \equiv 0$, since in that case the left-hand side vanishes from (1.17) and (1.18), while the right-hand side is nonnegative (it is not less than the integral of the norm square of $\left.\Delta x_{\alpha} u_{i}+2 \nabla u_{i} \cdot \nabla x_{\alpha}-2 \sum_{j=1}^{n} b_{i j}^{\alpha} u_{j}\right)$.

Putting (1.18) into (1.20), we have

$$
\begin{aligned}
\left(\lambda_{n+1}-\lambda_{i}\right) I_{\alpha i} & +\sum_{j=1}^{n}\left(\lambda_{i}-\lambda_{j}\right)\left(\lambda_{n+1}-\lambda_{j}\right)\left(a_{i j}^{\alpha}\right)^{2} \\
h f i l l & \leq(1+1 / \epsilon) \int_{\Omega}\left(\Delta x_{\alpha}\right)^{2} u_{i}^{2}+4(1+\epsilon) \int_{\Omega}\left|\nabla u_{i} \cdot \nabla x_{\alpha}\right|^{2} .
\end{aligned}
$$

By making summation of the terms of $\left(\lambda_{n+1}-\lambda_{i}\right) \times(1.21)$, we get

$$
\sum_{i=1}^{n}\left(\lambda_{n+1}-\lambda_{i}\right)^{2} I_{\alpha i}
$$

$h$ fill $\leq(1+1 / \epsilon) \sum_{i=1}^{n}\left(\lambda_{n+1}-\lambda_{i}\right) \int_{\Omega}\left(\Delta x_{\alpha}\right)^{2} u_{i}^{2}+4(1+\epsilon) \sum_{i=1}^{n}\left(\lambda_{n+1}-\lambda_{i}\right) \int_{\Omega}\left|\nabla u_{i} \cdot \nabla x_{\alpha}\right|^{2}$.

By use of the identities

$$
\begin{gathered}
\Delta x=-m \mathbf{H}, \quad \sum_{\alpha=1}^{N} I_{\alpha i}=\sum_{\alpha=1}^{N} \int_{\Omega}\left|\nabla x_{\alpha}\right|^{2} u_{i}^{2}=m \int_{\Omega} u_{i}^{2}=m, \\
\sum_{\alpha=1}^{N} \int_{\Omega}\left|\nabla u_{i} \cdot \nabla x_{\alpha}\right|^{2}=\int_{\Omega}\left|\nabla u_{i}\right|^{2}=-\int_{\Omega} u_{i} \Delta u_{i}=\int_{\Omega} u_{i}\left(\lambda_{i} u_{i}-V u_{i}\right)=\lambda_{i}-\int_{\Omega} V u_{i}^{2},
\end{gathered}
$$

and by making summation of (1.22) over $\alpha$ from 1 to $N$, we obtain

$$
\begin{aligned}
& m \sum_{i=1}^{n}\left(\lambda_{n+1}-\lambda_{i}\right)^{2} \\
\leq & (1+1 / \epsilon) m^{2} \sum_{i=1}^{n}\left(\lambda_{n+1}-\lambda_{i}\right) \int_{\Omega}|\mathbf{H}|^{2} u_{i}^{2}+4(1+\epsilon) \sum_{i=1}^{n}\left(\lambda_{n+1}-\lambda_{i}\right)\left(\lambda_{i}-\int_{\Omega} V u_{i}^{2}\right) .
\end{aligned}
$$

Writing

$$
|\mathbf{H}|_{\infty}^{2}=\sup _{\bar{\Omega}}|\mathbf{H}|^{2}, \quad V_{0}=\inf _{\bar{\Omega}} V
$$


we have from (1.23) and (1.24) for any real number $\epsilon>0$

$$
\begin{aligned}
& \sum_{i=1}^{n}\left(\lambda_{n+1}-\lambda_{i}\right)^{2} \\
& \quad \leq(1+1 / \epsilon) m^{2}|\mathbf{H}|_{\infty}^{2} \sum_{i=1}^{n}\left(\lambda_{n+1}-\lambda_{i}\right)+4(1+\epsilon) \sum_{i=1}^{n}\left(\lambda_{n+1}-\lambda_{i}\right)\left(\lambda_{i}-V_{0}\right) .
\end{aligned}
$$

Thus we have proved the following main result of this section:

TheOREM 1.1. Let $x: M \rightarrow R^{N}$ be an $m$-dimensional submanifold in $N$-dimensional Euclidean space. Let $\Omega \subset M$ be a bounded domain. Then the spectrum of the Schrödinger operator $L=\Delta-V$ satisfies the following inequality, for any real number $\epsilon>0$, and for any $n=1,2, \ldots$ :

$$
\begin{aligned}
& m \sum_{i=1}^{n}\left(\lambda_{n+1}-\lambda_{i}\right)^{2} \\
& \quad \leq(1+1 / \epsilon) m^{2}|\mathbf{H}|_{\infty}^{2} \sum_{i=1}^{n}\left(\lambda_{n+1}-\lambda_{i}\right)+4(1+\epsilon) \sum_{i=1}^{n}\left(\lambda_{n+1}-\lambda_{i}\right)\left(\lambda_{i}-V_{0}\right) .
\end{aligned}
$$

Writing

$$
\tilde{\lambda}_{n+1}=\lambda_{n+1}-V_{0}, \quad \tilde{\lambda}_{i}=\lambda_{i}-V_{0}, \quad i=1,2, \ldots, n,
$$

we have

$$
\tilde{\lambda}_{n} \geq \tilde{\lambda}_{n-1} \geq \cdots \geq \tilde{\lambda}_{2} \geq \tilde{\lambda}_{1}=\lambda_{1}-V_{0}=\frac{\int\left|\nabla u_{1}\right|^{2}}{\int u_{1}^{2}}+\frac{\int V u_{1}^{2}}{\int u_{1}^{2}}-V_{0} \geq \frac{\int\left|\nabla u_{1}\right|^{2}}{\int u_{1}^{2}}>0 .
$$

Then (1.26) can be rewritten as

$$
m \sum_{i=1}^{n}\left(\tilde{\lambda}_{n+1}-\tilde{\lambda}_{i}\right)^{2} \leq(1+1 / \epsilon) m^{2}|\mathbf{H}|_{\infty}^{2} \sum_{i=1}^{n}\left(\tilde{\lambda}_{n+1}-\tilde{\lambda}_{i}\right)+4(1+\epsilon) \sum_{i=1}^{n}\left(\tilde{\lambda}_{n+1}-\tilde{\lambda}_{i}\right) \tilde{\lambda}_{i} .
$$

It is easy to see that $(1.26)^{\prime}$ is equivalent to

$$
\begin{aligned}
\tilde{\lambda}_{n+1} \leq & \frac{1}{2} m(1+1 / \epsilon)|\mathbf{H}|_{\infty}^{2}+\frac{1}{n}\left(1+\frac{2}{m}(1+\epsilon)\right) \sum_{i=1}^{n} \tilde{\lambda}_{i} \\
& +\frac{1}{2 n}\left\{\left[(1+1 / \epsilon) m n|\mathbf{H}|_{\infty}^{2}+\left(2+\frac{4}{m}(1+\epsilon)\right) \sum_{i=1}^{n} \tilde{\lambda}_{i}\right]^{2}\right. \\
& \left.-4 n\left(1+\frac{4}{m}(1+\epsilon)\right) \sum_{i=1}^{n} \tilde{\lambda}_{i}^{2}-4 m n(1+1 / \epsilon)|\mathbf{H}|_{\infty}^{2} \sum_{i=1}^{n} \tilde{\lambda}_{i}\right\}^{\frac{1}{2}} .
\end{aligned}
$$

Noting $\left(\sum_{i=1}^{n} \tilde{\lambda}_{i}\right)^{2} \leq n \sum_{i=1}^{n} \tilde{\lambda}_{i}^{2}$, we have from $(1.27)$

$$
\tilde{\lambda}_{n+1} \leq m(1+1 / \epsilon)|\mathbf{H}|_{\infty}^{2}+\frac{1}{n}\left(1+\frac{4}{m}(1+\epsilon)\right) \sum_{i=1}^{n} \tilde{\lambda}_{i}
$$

Thus we have proved the following weak, but simpler version of Theorem 1.1:

TheOREm 1.2. Let $x: M \rightarrow R^{N}$ be an m-dimensional submanifold in $N$-dimensional Euclidean space. Let $\Omega \subset M$ be a bounded domain. Then the spectrum of the Schrödinger 
operator $L=\Delta-V$ satisfies the following inequality, for any real number $\epsilon>0$ :

$$
\lambda_{n+1}-V_{0} \leq m(1+1 / \epsilon)|\mathbf{H}|_{\infty}^{2}+\frac{1}{n}\left(1+\frac{4}{m}(1+\epsilon)\right) \sum_{i=1}^{n}\left(\lambda_{i}-V_{0}\right), \quad n=1,2, \ldots
$$

Now we prove that Theorem 1.2 implies the following conclusion:

TheOREM 1.3. Let $x: M \rightarrow R^{N}$ be an $m$-dimensional submanifold in $N$-dimensional Euclidean space. Let $\Omega \subset M$ be a bounded domain. Then the spectrum of the Schrödinger operator $L=\Delta-V$ satisfies the following inequality, for any real number $\epsilon>0$, and for any $n \in N$ satisfying $\lambda_{n+1}>\lambda_{n}$ :

$$
\sum_{i=1}^{n} \frac{4(1+\epsilon)\left(\lambda_{i}-V_{0}\right)+(1+1 / \epsilon) m^{2}|\mathbf{H}|_{\infty}^{2}}{\lambda_{n+1}-\lambda_{i}} \geq m n, \quad n=1,2, \ldots
$$

and equivalently

$$
\sum_{i=1}^{n} \frac{4(1+\epsilon) \tilde{\lambda}_{i}+(1+1 / \epsilon) m^{2}|\mathbf{H}|_{\infty}^{2}}{\tilde{\lambda}_{n+1}-\tilde{\lambda}_{i}} \geq m n, \quad n=1,2, \ldots
$$

Proof. (1.28) or $(1.28)^{\prime}$ can be rewritten as

$$
\frac{1}{m n}\left[4(1+\epsilon) \sum_{i=1}^{n} \tilde{\lambda}_{i}+m^{2} n(1+1 / \epsilon)|\mathbf{H}|_{\infty}^{2}\right] \geq \tilde{\lambda}_{n+1}-\frac{1}{n} \sum_{i=1}^{n} \tilde{\lambda}_{i},
$$

that is,

$$
m n \leq \frac{4(1+\epsilon) \sum_{i=1}^{n} \tilde{\lambda}_{i}+m^{2} n(1+1 / \epsilon)|\mathbf{H}|_{\infty}^{2}}{\tilde{\lambda}_{n+1}-\frac{1}{n} \sum_{i=1}^{n} \tilde{\lambda}_{i}}
$$

From $(1.29)$ and $(1.30)^{\prime}$, we only need to prove

$$
\frac{4(1+\epsilon) \sum_{i=1}^{n} \tilde{\lambda}_{i}+m^{2} n(1+1 / \epsilon)|\mathbf{H}|_{\infty}^{2}}{\tilde{\lambda}_{n+1}-\frac{1}{n} \sum_{i=1}^{n} \tilde{\lambda}_{i}} \leq \sum_{i=1}^{n} \frac{4(1+\epsilon) \tilde{\lambda}_{i}+(1+1 / \epsilon) m^{2}|\mathbf{H}|_{\infty}^{2}}{\tilde{\lambda}_{n+1}-\tilde{\lambda}_{i}}
$$

We consider the function

$$
\begin{aligned}
f(x) & =\frac{4(1+\epsilon) x+(1+1 / \epsilon) m^{2}|\mathbf{H}|_{\infty}^{2}}{\tilde{\lambda}_{n+1}-x} \\
& =-4(1+\epsilon)+\frac{(1+1 / \epsilon) m^{2}|\mathbf{H}|_{\infty}^{2}+4(1+\epsilon) \tilde{\lambda}_{n+1}}{\tilde{\lambda}_{n+1}-x} .
\end{aligned}
$$

It is convex when $x<\tilde{\lambda}_{n+1}$. Thus

$$
f\left(\frac{\tilde{\lambda}_{1}+\tilde{\lambda}_{2}+\cdots+\tilde{\lambda}_{n}}{n}\right) \leq \frac{1}{n}\left[f\left(\tilde{\lambda}_{1}\right)+f\left(\tilde{\lambda}_{2}\right)+\cdots+f\left(\tilde{\lambda}_{n}\right)\right] .
$$

It is easy to check that (1.32) is equivalent to (1.31). Thus we have completed the proof of Theorem 1.3.

Theorem 1.3 has the following corollary:

Theorem 1.4. Let $x: M \rightarrow R^{N}$ be an m-dimensional submanifold in $N$-dimensional Euclidean space. Let $\Omega \subset M$ be a bounded domain. Then the spectrum of the Schrödinger 
operator $L=\Delta-V$ satisfies the following inequality, for any real number $\epsilon>0$ :

$$
\lambda_{n+1}-\lambda_{n} \leq \frac{4(1+\epsilon)}{m n} \sum_{i=1}^{n}\left(\lambda_{i}-V_{0}\right)+(1+1 / \epsilon) m|\mathbf{H}|_{\infty}^{2}, \quad n=1,2, \ldots
$$

and equivalently

$$
\tilde{\lambda}_{n+1}-\tilde{\lambda}_{n} \leq \frac{4(1+\epsilon)}{m n} \sum_{i=1}^{n} \tilde{\lambda}_{i}+(1+1 / \epsilon) m|\mathbf{H}|_{\infty}^{2}, \quad n=1,2, \ldots
$$

Proof. Replacing the $\lambda_{i}$ in the denominator of the left-hand side of (1.29) by $\lambda_{n}$, we obtain (1.33).

REMARK 1.1. If $x: M \rightarrow R^{N}$ is an $m$-dimensional minimal submanifold and $V \equiv 0$, letting $\epsilon \rightarrow 0$ in Theorem 1.4, we recover S. Y. Cheng's result [2].

When $M=R^{m}$ and $V \equiv 0$, in this case $x: M \rightarrow R^{N}$ is an $m$-dimensional totally geodesic submanifold, we have $H \equiv 0$. We have, from Theorems 1.1 to 1.4 by letting $\epsilon \rightarrow 0$ :

Corollary 1.1 (Yang [8]). Let $\Omega \subset R^{m}$ be a bounded domain. Then the spectrum of the Laplacian $\Delta$ satisfies the inequality

$$
m \sum_{i=1}^{n}\left(\lambda_{n+1}-\lambda_{i}\right)^{2} \leq 4 \sum_{i=1}^{n}\left(\lambda_{n+1}-\lambda_{i}\right) \lambda_{i}, \quad n=1,2, \ldots
$$

Corollary 1.2 (Ashbaugh [1], Yang [8]). Let $\Omega \subset R^{m}$ be a bounded domain. Then the spectrum of the Laplacian $\Delta$ satisfies the inequality

$$
\lambda_{n+1} \leq \frac{1}{n}\left(1+\frac{4}{m}\right) \sum_{i=1}^{n} \lambda_{i}, \quad n=1,2, \ldots
$$

Corollary 1.3 (Hile-Protter [3]). Let $\Omega \subset R^{m}$ be a bounded domain. Then the spectrum of the Laplacian $\Delta$ satisfies the inequality

$$
4 \sum_{i=1}^{n} \frac{\lambda_{i}}{\lambda_{n+1}-\lambda_{i}} \geq m n, \quad n=1,2, \ldots
$$

Corollary 1.4 (Payne-Pólya-Weinberger [5], Thompson [7]). Let $\Omega \subset R^{m}$ be a bounded domain. Then the spectrum of the Laplacian $\Delta$ satisfies the inequality

$$
\lambda_{n+1}-\lambda_{n} \leq \frac{4}{m n} \sum_{i=1}^{n} \lambda_{i}, \quad n=1,2, \ldots
$$

2. Minimal submanifold in the unit sphere. In this section, let $x: M \rightarrow S^{N-1}(1)$ be an $m$-dimensional closed (i.e., compact without boundary) minimal submanifold in the Euclidean unit sphere. As in the previous section, we consider the Schrödinger operator

$$
L=\Delta-V,
$$

where $V$ is a nonnegative smooth function on $M$. We shall consider the following Dirichlet eigenvalue problem for the Schrödinger operator $L$ on $M$ :

$$
L u=-\lambda u \quad \text { on } \quad M .
$$


As is well-known, the eigenvalues $\left\{\lambda_{k}\right\}$ of (2.2) are nonnegative, and can be arranged in nondecreasing order as follows:

$$
0 \leq \lambda_{1} \leq \lambda_{2} \leq \cdots \leq \lambda_{n} \leq \cdots
$$

Let $x=\left(x_{1}, \ldots, x_{N}\right): M \rightarrow S^{N-1}(1) \subset R^{N}$ be the minimal immersion. Let $\left\{\left(u_{i}, \lambda_{i}\right)\right\}_{i=1, \ldots, n}$ be the normalized first $n$ eigenfunctions and their corresponding eigenvalues. Then

$$
\begin{gathered}
\Delta x_{\alpha}=-m x_{\alpha}, \quad \sum_{\alpha=1}^{N} x_{\alpha}^{2}=1, \\
L u_{i}=-\lambda_{i} u_{i}, \quad i=1, \ldots, n .
\end{gathered}
$$

Noting that all formulas from preceding section still hold, we have from (1.13) and (1.17) by use of $(2.3)$

$$
\begin{aligned}
& \left(\lambda_{n+1}-\lambda_{i}\right) \int_{M}\left(\phi_{i}^{\alpha}\right)^{2} \cdot\left[I_{\alpha i}+\sum_{j=1}^{n}\left(\lambda_{i}-\lambda_{j}\right)\left(a_{i j}^{\alpha}\right)^{2}\right] \\
\leq & \int_{M}\left(\phi_{i}^{\alpha}\right)^{2} \cdot\left[\int_{M}\left(\Delta x_{\alpha} u_{i}+2 \nabla u_{i} \cdot \nabla x_{\alpha}\right)^{2}-4 \sum_{j=1}^{n}\left(b_{i j}^{\alpha}\right)^{2}\right] \\
= & \int_{M}\left(\phi_{i}^{\alpha}\right)^{2} \cdot\left[\int_{M}\left(\Delta x_{\alpha}\right)^{2} u_{i}^{2}+4 \int_{M}\left|\nabla u_{i} \cdot \nabla x_{\alpha}\right|^{2}-2 m \int_{M} u_{i} \nabla u_{i} \cdot \nabla x_{\alpha}^{2}-4 \sum_{j=1}^{n}\left(b_{i j}^{\alpha}\right)^{2}\right] \\
= & \int_{M}\left(\phi_{i}^{\alpha}\right)^{2} \cdot\left[\int_{M} m^{2} x_{\alpha}^{2} u_{i}^{2}+4 \int_{M}\left|\nabla u_{i} \cdot \nabla x_{\alpha}\right|^{2}-2 m \int_{M} u_{i} \nabla u_{i} \cdot \nabla x_{\alpha}^{2}-4 \sum_{j=1}^{n}\left(b_{i j}^{\alpha}\right)^{2}\right],
\end{aligned}
$$

thus we get

$$
\begin{aligned}
& \left(\lambda_{n+1}-\lambda_{i}\right)\left[I_{\alpha i}+\sum_{j=1}^{n}\left(\lambda_{i}-\lambda_{j}\right)\left(a_{i j}^{\alpha}\right)^{2}\right] \\
& \quad \leq \int_{M} m^{2} x_{\alpha}^{2} u_{i}^{2}+4 \int_{M}\left|\nabla u_{i} \cdot \nabla x_{\alpha}\right|^{2}-2 m \int_{M} u_{i} \nabla u_{i} \cdot \nabla x_{\alpha}^{2}-4 \sum_{j=1}^{n}\left(b_{i j}^{\alpha}\right)^{2} .
\end{aligned}
$$

Putting (1.18) into (2.6), we have

$$
\begin{aligned}
& \left(\lambda_{n+1}-\lambda_{i}\right) I_{\alpha i}+\sum_{j=1}^{n}\left(\lambda_{i}-\lambda_{j}\right)\left(\lambda_{n+1}-\lambda_{j}\right)\left(a_{i j}^{\alpha}\right)^{2} \\
& \quad \leq \int_{M} m^{2} x_{\alpha}^{2} u_{i}^{2}+4 \int_{M}\left|\nabla u_{i} \cdot \nabla x_{\alpha}\right|^{2}-2 m \int_{M} u_{i} \nabla u_{i} \cdot \nabla x_{\alpha}^{2} .
\end{aligned}
$$

By making summation of the terms of $\left(\lambda_{n+1}-\lambda_{i}\right) \times(2.7)$, we get

$$
\begin{aligned}
\sum_{i=1}^{n}\left(\lambda_{n+1}-\right. & \left.\lambda_{i}\right)^{2} I_{\alpha i} \\
\leq & \sum_{i=1}^{n}\left(\lambda_{n+1}-\lambda_{i}\right) \int_{M} m^{2} x_{\alpha}^{2} u_{i}^{2}+4 \sum_{i=1}^{n}\left(\lambda_{n+1}-\lambda_{i}\right) \int_{M}\left|\nabla u_{i} \cdot \nabla x_{\alpha}\right|^{2} \\
& -2 m \sum_{i=1}^{n}\left(\lambda_{n+1}-\lambda_{i}\right) \int_{M} u_{i} \nabla u_{i} \cdot \nabla x_{\alpha}^{2} .
\end{aligned}
$$


By use of the identities

$$
\begin{aligned}
V_{0}=\inf _{\bar{\Omega}} V, \quad \sum_{\alpha=1}^{N} I_{\alpha i} & =\sum_{\alpha=1}^{N} \int_{M}\left|\nabla x_{\alpha}\right|^{2} u_{i}^{2}=m \int_{M} u_{i}^{2}=m, \\
\sum_{\alpha=1}^{N} \int_{M}\left|\nabla u_{i} \cdot \nabla x_{\alpha}\right|^{2} & =\int_{M}\left|\nabla u_{i}\right|^{2}=-\int_{M} u_{i} \Delta u_{i} \\
& =\int_{M} u_{i}\left(\lambda_{i} u_{i}-V u_{i}\right)=\lambda_{i}-\int_{M} V u_{i}^{2},
\end{aligned}
$$

and by making summation of (2.8) over $\alpha$ from 1 to $N$ and using (2.3), we get

$$
m \sum_{i=1}^{n}\left(\lambda_{n+1}-\lambda_{i}\right)^{2} \leq m^{2} \sum_{i=1}^{n}\left(\lambda_{n+1}-\lambda_{i}\right)+4 \sum_{i=1}^{n}\left(\lambda_{n+1}-\lambda_{i}\right)\left(\lambda_{i}-V_{0}\right) .
$$

Thus we have proved the following

THEOREM 2.1 Let $x: M \rightarrow S^{N-1}(1)$ be an m-dimensional closed minimal submanifold in an $(N-1)$-dimensional Euclidean sphere. Let $V$ be a nonnegative function on $M$. Then the spectrum of the Schrödinger operator $L=\Delta-V$ on $M$ satisfies the inequality

$$
m \sum_{i=1}^{n}\left(\lambda_{n+1}-\lambda_{i}\right)^{2} \leq m^{2} \sum_{i=1}^{n}\left(\lambda_{n+1}-\lambda_{i}\right)+4 \sum_{i=1}^{n}\left(\lambda_{n+1}-\lambda_{i}\right)\left(\lambda_{i}-V_{0}\right), \quad n=1,2, \ldots
$$

Writing

$$
\tilde{\lambda}_{n+1}=\lambda_{n+1}-V_{0}, \quad \tilde{\lambda}_{i}=\lambda_{i}-V_{0}, \quad i=1,2, \ldots, n,
$$

we have

$$
\tilde{\lambda}_{n+1} \geq \tilde{\lambda}_{n} \geq \cdots \geq \tilde{\lambda}_{1} \geq 0
$$

Then (2.9) can be rewritten as

$$
\sum_{i=1}^{n}\left(\tilde{\lambda}_{n+1}-\tilde{\lambda}_{i}\right)^{2} \leq m^{2} \sum_{i=1}^{n}\left(\tilde{\lambda}_{n+1}-\tilde{\lambda}_{i}\right)+4 \sum_{i=1}^{n}\left(\tilde{\lambda}_{n+1}-\tilde{\lambda}_{i}\right) \tilde{\lambda}_{i}
$$

It is easy to see that $(2.9)^{\prime}$ is equivalent to

$$
\begin{aligned}
\tilde{\lambda}_{n+1} \leq & \frac{1}{2} m+\frac{1}{n}(1+2 / m) \sum_{i=1}^{n} \tilde{\lambda}_{i} \\
& +\frac{1}{2 n}\left\{\left[m n+(2+4 / m) \sum_{i=1}^{n} \tilde{\lambda}_{i}\right]^{2}-4 n(1+4 / m) \sum_{i=1}^{n} \tilde{\lambda}_{i}^{2}-4 m n \sum_{i=1}^{n} \tilde{\lambda}_{i}\right\}^{\frac{1}{2}} .
\end{aligned}
$$

Noting $\left(\sum_{i=1}^{n} \tilde{\lambda}_{i}\right)^{2} \leq n \sum_{i=1}^{n} \tilde{\lambda}_{i}^{2}$, we have from $(2.10)$

$$
\tilde{\lambda}_{n+1} \leq m+\frac{1}{n}(1+4 / m) \sum_{i=1}^{n} \tilde{\lambda}_{i} .
$$

Thus we proved the following weak, but simpler version of Theorem 2.1:

TheOREm 2.2. Let $x: M \rightarrow S^{N-1}(1)$ be an $m$-dimensional closed minimal submanifold in an $(N-1)$-dimensional Euclidean sphere. Let $V$ be a nonnegative function on $M$. 
Then the spectrum of the Schrödinger operator $L=\Delta-V$ on $M$ satisfies the inequality

$$
\lambda_{n+1}-V_{0} \leq m+\frac{1}{n}(1+4 / m) \sum_{i=1}^{n}\left(\lambda_{i}-V_{0}\right), \quad n=1,2, \ldots
$$

Now we prove that Theorem 2.2 implies the following conclusion:

TheOREM 2.3. Let $x: M \rightarrow S^{N-1}(1)$ be an $m$-dimensional closed minimal submanifold in an $(N-1)$-dimensional Euclidean sphere. Let $V$ be a nonnegative function on $M$. Then the spectrum of the Schrödinger operator $L=\Delta-V$ on $M$ satisfies the following inequality, for any $n \in N$ satisfying $\lambda_{n+1}>\lambda_{n}$ :

$$
\sum_{i=1}^{n} \frac{m^{2}+4\left(\lambda_{i}-V_{0}\right)}{\lambda_{n+1}-\lambda_{i}} \geq m n, \quad n=1,2, \ldots
$$

and equivalently

$$
\sum_{i=1}^{n} \frac{m^{2}+4 \tilde{\lambda}_{i}}{\tilde{\lambda}_{n+1}-\tilde{\lambda}_{i}} \geq m n, \quad n=1,2, \ldots
$$

Proof. (2.11) or $(2.11)^{\prime}$ can be rewritten as

$$
\frac{1}{m n} \geq \frac{\tilde{\lambda}_{n+1}-\frac{1}{n} \sum_{i=1}^{n} \tilde{\lambda}_{i}}{m^{2} n+4 \sum_{i=1}^{n} \tilde{\lambda}_{i}}
$$

that is,

$$
m n \leq \frac{m^{2} n+4 \sum_{i=1}^{n} \tilde{\lambda}_{i}}{\tilde{\lambda}_{n+1}-\frac{1}{n} \sum_{i=1}^{n} \tilde{\lambda}_{i}}
$$

From $(2.12)^{\prime}$ and (2.13), we only need to prove

$$
\frac{m^{2} n+4 \sum_{i=1}^{n} \tilde{\lambda}_{i}}{\tilde{\lambda}_{n+1}-\frac{1}{n} \sum_{i=1}^{n} \tilde{\lambda}_{i}} \leq \sum_{i=1}^{n} \frac{m^{2}+4 \tilde{\lambda}_{i}}{\tilde{\lambda}_{n+1}-\tilde{\lambda}_{i}}
$$

Consider the function

$$
f(x)=\frac{m^{2}+4 x}{\tilde{\lambda}_{n+1}-x}=-4+\frac{m^{2}+4 \tilde{\lambda}_{n+1}}{\tilde{\lambda}_{n+1}-x} .
$$

It is convex when $x<\tilde{\lambda}_{n+1}$. Thus

$$
f\left(\frac{\tilde{\lambda}_{1}+\tilde{\lambda}_{2}+\cdots+\tilde{\lambda}_{n}}{n}\right) \leq \frac{1}{n}\left[f\left(\tilde{\lambda}_{1}\right)+f\left(\tilde{\lambda}_{2}\right)+\cdots+f\left(\tilde{\lambda}_{n}\right)\right] .
$$

It is easy to check that (2.15) is equivalent to (2.14). Therefore the proof of Theorem 2.3 is complete.

Theorem 2.3 has the following corollary:

THEOREM 2.4. Let $x: M \rightarrow S^{N-1}(1)$ be an $m$-dimensional closed minimal submanifold in an $(N-1)$-dimensional Euclidean sphere. Let $V$ be a nonnegative function on $M$. Then the spectrum of the Schrödinger operator $L=\Delta-V$ on $M$ satisfies the inequality

$$
\lambda_{n+1}-\lambda_{n} \leq m+\frac{4}{m n} \sum_{i=1}^{n}\left(\lambda_{i}-V_{0}\right), \quad n=1,2, \ldots
$$


and equivalently

$$
\tilde{\lambda}_{n+1}-\tilde{\lambda}_{n} \leq m+\frac{4}{m n} \sum_{i=1}^{n} \tilde{\lambda}_{i}, \quad n=1,2, \ldots
$$

Proof. Replacing the $\lambda_{i}$ by $\lambda_{n}$ in the denominator of the left-hand side of (2.12), we obtain (2.16).

In the case $V \equiv 0$ the first eigenvalue is zero, and it is traditional to reindex so that $\lambda_{1}$ is the first nonzero eigenvalue. We indicate this by denoting the eigenvalues by $\lambda_{j}^{\prime}$, that is,

$$
\lambda_{0}^{\prime}=\lambda_{1}=0, \lambda_{1}^{\prime}=\lambda_{2}, \ldots, \lambda_{j}^{\prime}=\lambda_{j+1}, \ldots, \lambda_{n}^{\prime}=\lambda_{n+1} .
$$

In this case, from Theorems 2.1 to 2.4 , we have

Corollary 2.1. Let $x: M \rightarrow S^{N-1}(1)$ be an m-dimensional closed minimal submanifold in an $(N-1)$-dimensional Euclidean sphere. Then the spectrum of the Laplacian $\Delta$ on $M$ satisfies the inequality

$$
m \sum_{j=0}^{n-1}\left(\lambda_{n}^{\prime}-\lambda_{j}^{\prime}\right)^{2} \leq m^{2} \sum_{j=0}^{n-1}\left(\lambda_{n}^{\prime}-\lambda_{j}^{\prime}\right)+4 \sum_{j=0}^{n-1}\left(\lambda_{n}^{\prime}-\lambda_{j}^{\prime}\right) \lambda_{j}^{\prime}, \quad n=2,3, \ldots
$$

Corollary 2.2. Let $x: M \rightarrow S^{N-1}(1)$ be an m-dimensional closed minimal submanifold in an $(N-1)$-dimensional Euclidean sphere. Then the spectrum of the Laplacian $\Delta$ on $M$ satisfies the inequality

$$
\lambda_{n}^{\prime} \leq m+\frac{1}{n}(1+4 / m) \sum_{j=0}^{n-1} \lambda_{j}^{\prime}, \quad n=2,3, \ldots
$$

Corollary 2.3. Let $x: M \rightarrow S^{N-1}(1)$ be an m-dimensional closed minimal submanifold in an $(N-1)$-dimensional Euclidean sphere. Then the spectrum of the Laplacian $\Delta$ on $M$ satisfies the following inequality, for any $n \in N$ satisfying $\lambda_{n}^{\prime}>\lambda_{n-1}^{\prime}$ :

$$
\sum_{j=0}^{n-1} \frac{m^{2}+4 \lambda_{j}^{\prime}}{\lambda_{n}^{\prime}-\lambda_{j}^{\prime}} \geq m n, \quad n=2,3, \ldots
$$

Corollary 2.4. Let $x: M \rightarrow S^{N-1}(1)$ be an m-dimensional closed minimal submanifold in an $(N-1)$-dimensional Euclidean sphere. Then the spectrum of the Laplacian $\Delta$ on $M$ satisfies the inequality

$$
\lambda_{n}^{\prime}-\lambda_{n-1}^{\prime} \leq m+\frac{4}{m n} \sum_{j=0}^{n-1} \lambda_{j}^{\prime}, \quad n=2,3, \ldots
$$

Noting $\lambda_{0}^{\prime}=0$, we immediately get the following Yang-Yau's result from Corollary 2.4. Corollary 2.5 (Leung [4], Yang-Yau [9]). Let $x: M \rightarrow S^{N-1}(1)$ be an $m$-dimensional closed minimal submanifold in an $(N-1)$-dimensional Euclidean sphere. Then the spectrum of the Laplacian $\Delta$ on $M$ satisfies the inequality

$$
\lambda_{n}^{\prime}-\lambda_{n-1}^{\prime} \leq m+\frac{2}{m n}\left(\sum_{j=1}^{n-1} \lambda_{j}^{\prime}+\sqrt{\left(\sum_{j=1}^{n-1} \lambda_{j}^{\prime}\right)^{2}+m^{2} n \sum_{j=1}^{n-1} \lambda_{j}^{\prime}} .\right.
$$


Acknowledgements. The first author began this research during his stay at the Institute of Mathematics of TU Berlin as an AVH fellow in 2002. He would like to express his thanks to Prof. Dr. Udo Simon and Dr. Martin Wiehe for their help. We would also like to thank the referee for some helpful comments.

\section{References}

[1] M. S. Ashbaugh, Isoperimetric and universal inequalities for eigenvalues, in: Spectral Theory and Geometry, E. B. Davies and Yu. Safarov (eds.), London Math. Soc. Lecture Note Series 273, Cambridge University Press, 1999, 95-139.

[2] S. Y. Cheng, Eigenfunctions and eigenvalues of Laplacian, Differential Geometry, S. S. Chern and R. Osserman (eds.), Proc. Symp. Pure Math. 27, Part 2, Amer. Math. Soc., Providence, Rhode Island, 1975, 185-193.

[3] G. N. Hile and M. H. Protter, Inequalities for eigenvalues of the Laplacian, Indiana Univ. Math. J. 29 (1980), 523-538.

[4] P. F. Leung, On the consecutive eigenvalues of the Laplacian of a compact minimal submanifold in a sphere, J. Austral. Math. Soc. (Series A) 50 (1991), 409-416.

[5] L. E. Payne, G. Pólya and H. F. Weinberger, On the ratio of consecutive eigenvalues, J. Math. Physics 35 (1956), 289-298.

[6] R. Scheon and S.-T. Yau, Lectures on Differential Geometry, Conference Proceedings and Lecture Notes in Geometry and Topology, Vol. 1, International Press, Cambridge, MA, 1994.

[7] C. J. Thompson, On the ratio of consecutive eigenvalues in $n$-dimensions, Stud. Appl. Math. 48 (1969), 281-283.

[8] H. C. Yang, Estimates of the difference between consecutive eigenvalues, preprint, 1995 (revision of Internat. Center for Theoretical Physics Preprint IC/91/60, Trieste, Italy, April, 1991).

[9] P. C. Yang and S.-T. Yau, Eigenvalues of the Laplacian of compact Riemann surfaces and minimal submanifolds, Ann. Scuola Norm. Sup. Pisa Cl. Sci. (4) 7 (1980), 55-63. 